Braz J Med Biol Res, August 2012, Volume 45(8) 746-752

doi: 10.1590/S0100-879X2012007500077

Cloning, bioinformatics analysis, and expression of the dust mite allergen Der 5 of Dermatophagoides farinae

Yubao Cui, Ying Zhou, Guifang Ma, Li Yang, Yungang Wang and Weihong Shi

The Brazilian Journal of Medical and Biological Research is partially financed by

\section{Q}

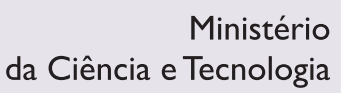

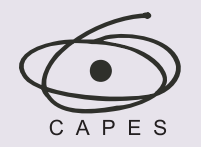

Ministério da Educação
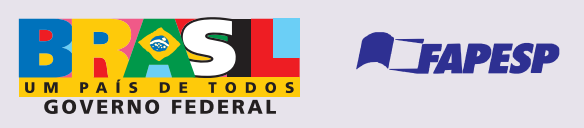

Institutional Sponsors

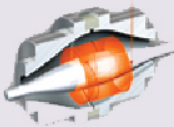

$\oplus$ SHIMADZU UNICAMP
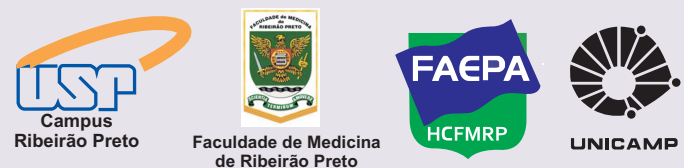


\title{
Cloning, bioinformatics analysis, and expression of the dust mite allergen Der $f 5$ of Dermatophagoides farinae
}

\author{
Yubao Cui, Ying Zhou, Guifang Ma, Li Yang, Yungang Wang \\ and Weihong Shi \\ Department of Laboratory Medicine, Yancheng Health Vocational and Technical College, \\ Jiangsu Yancheng, China
}

\begin{abstract}
Crude extracts of house dust mites are used clinically for diagnosis and immunotherapy of allergic diseases, including bronchial asthma, perennial rhinitis, and atopic dermatitis. However, crude extracts are complexes with non-allergenic antigens and lack effective concentrations of important allergens, resulting in several side effects. Dermatophagoides farinae (Hughes; Acari: Pyroglyphidae) is one of the predominant sources of dust mite allergens, which has more than 30 groups of allergen. The cDNA coding for the group 5 allergen of $D$. farinae from China was cloned, sequenced and expressed. According to alignment using the VECTOR NTI 9.0 software, there were eight mismatched nucleotides in five cDNA clones resulting in seven incompatible amino acid residues, suggesting that the Der $f 5$ allergen might have sequence polymorphism. Bioinformatics analysis revealed that the matured Der $f 5$ allergen has a molecular mass of $13604.03 \mathrm{Da}$, a theoretical pl of 5.43 and is probably hydrophobic and cytoplasmic. Similarities in amino acid sequences between Der $\mathrm{f} 5$ and allergens of other domestic mite species, viz. Der p 5, Blo t 5, Sui m 5, and Lep d 5, were 79, 48, 53, and 37\%, respectively. Phylogenetic analysis indicated that Der 5 and Der p 5 clustered together. Blo 5 and Ale o 5 also clustered together, although Blomia tropicalis and Aleuroglyphus ovatus belong to different mite families, viz. Echimyopodidae and Acaridae, respectively.
\end{abstract}

Key words: Dermatophagoides farinae (Hughes); Der f 5; Cloning; Expression; Bioinformatics

\section{Introduction}

The dust mites, which are mostly represented by Dermatophagoides spp (Acari: Pyroglyphidae), are the major sources of indoor allergens associated with the occurrence of asthma, rhinitis, dermatitis, and other allergic diseases throughout the world (1-3). The investigation of crude dust mite extracts has demonstrated that more than 30 groups of components can induce IgE antibodies, and groups 1 and 2 were reported for the major allergens that reacted with $80 \%$ of sera from mite-sensitive patients $(4,5)$. Of the 23 groups of dust mite allergens listed in the International Union of Immunological Societies (IUIS) nomenclature dataset (http://www.allergen.org/), 21 have been identified from Dermatophagoides spp.

Currently, crude dust mite extracts are used for the diagnosis and therapy of mite-allergic patients. The dozens of complex components in these extracts - such as allergens, non-allergic, or toxic protein, and some enzymes - are postulated to be responsible for a variety of local and systemic pathological effects (6-8). To develop new diagnostic reagents and therapeutic strategies, a few groups of dust mite allergens have been cloned and expressed as genetic products, which were demonstrated to be more effective than their natural products $(9,10)$.

Polymorphisms have been described in several dust mite allergens in various regions. This could influence their biological effectiveness in diagnostics and immunotherapy (11). Thus, it is necessary to characterize dust mite allergens and establish a high expression system to produce these allergens on a large scale. Group 5 allergens were listed in $30 \%$ of mid-potency dust mite allergens (12). A large amount of these allergens is necessary for therapeutic purposes as well as for diagnosis. Here, we report the cloning, sequencing and expression in Escherichia coli of Der $f 5$ cDNA from $D$. farinae isolated in China.

Correspondence: Yubao Cui, Department of Laboratory Medicine, Yancheng Health Vocational and Technical College, Jiefangnan Road, 263, Yancheng 224006, Jiangsu Province, China. E-mail: ybcui1975@hotmail.com

Received December 5, 2011. Accepted May 3, 2012. Available online May 18, 2012. Published August 3, 2012. 


\section{Material and Methods}

\section{Preparation of Der $f 5$ cDNA and polymerase chain reaction (PCR)}

As described in previous reports (13-16), D. farinae isolated from the floors of rice and flour shops in Shanghai City, China, were cultured and isolated. About 600 mites were chosen for total RNA isolation by using an RNA isolator (Code D312; TaKaRa Biotech, China) and stored at $-80^{\circ} \mathrm{C}$. Based on the published sequence of Der $\mathrm{f} 5$ (GenBankAY 283283), nucleic acid primers were designed and synthesized; the forward primer was 5'-GGATCCAT GAAATTCATCATTGCTAT-3' and the reverse primer was 5'-CTCGAGTCAAACTTCAATCTTTTTAACACG-3', with BamHI and Xhol sites, respectively, at their 5'end (underlined). First, reverse transcription (RT) was performed using the total RNA isolated from mites with the High Fidelity PrimeScript ${ }^{\text {TM }}$ RT-PCR Kit (DR027; TaKaRa Biotech) in the PCR Thermal Cycler Dice (TP600; TaKaRa Biotech). Second, the RT product was used as the template for PCR in the same thermal cycler with PrimeSTAR ${ }^{\circledR}$ HS DNA Polymerase (DR010A; TaKaRa Biotech). Finally, $5 \mu \mathrm{L}$ of the PCR product was analyzed by $1.0 \%$ agarose gel electrophoresis and visualized with ImageMaster ${ }^{\circledR}$ VDS.

\section{Cloning and DNA sequencing}

Amplified PCR products were purified by gel electrophoresis on $1 \%$ low melting agarose gel. After the DNA was recovered with the Agarose Gel DNA Purification Kit 2.0 (DV805; TaKaRa Biotech), the purified DNA was added to a poly 'A' tail with a DNA A-Tailing Kit (D404; TaKaRa Biotech) and linked to the pMD19-T simple vector (D104; TaKaRa Biotech). The recombinant plasmid pMD19-T-Der $f$ 5 was then transformed into E. coli JM109 (D9052; TaKaRa Biotech). The positive clones were screened by blue-white selection on Luria-Bertani (LB) plates containing $100 \mu \mathrm{g} /$ $\mathrm{mL}$ ampicillin, and confirmed by restriction enzyme analysis with $\mathrm{BamHI}$ and $\mathrm{Xhol}$. DNA sequences were determined using an ABI PRISM ${ }^{\text {TM }}$ 377XL DNA Sequencer (TaKaRa Biotech).

\section{Construction of expression plasmids}

After sequencing, the recombinant plasmid was subjected to restriction enzyme digestion to release the Der $f$ 5 fragment. At the same time, pET28a(+) (Novagen, Kit Lot No. N72770, USA) was digested with BamHI and Xhol. The recovered Der 5 cDNA fragment was then sub-cloned into the recovered fragment from the digested expression vector pET28a(+) to create pET28a(+)-Der f 5 using the DNA Ligation Kit (D6023; TaKaRa Biotech). The E. colicompetent cells JM109 (D9052; TaKaRa Biotech) were transformed with pET28a(+)-Der $f 5$ plasmids, and positive clones were selected by blue-white screening and characterized by restriction enzyme analysis with $\mathrm{BamHI}$ and Xhol.

\section{Expression of recombinant Der $f 5$ (rDer $f 5)$ in E. coli BL21 (DE3)}

A $0.5-\mu L$ amount of the pET28a-(+)-Der $f 5$ plasmid was prepared using the MiniBEST Plasmid Purification Kit 2.0 (DV801A; TaKaRa Biotech) and used to transform 100 $\mu \mathrm{L}$ E. coli BL21 (DE3, Stratagene, USA). The E. coli BL21 carrying pET28a-(+)-Der $f 5$ was grown on LB plates containing $50 \mu \mathrm{g} / \mathrm{mL}$ kanamycin at $37^{\circ} \mathrm{C}$ overnight. The single colony was inoculated into $5 \mathrm{~mL}$ LB containing kanamycin and then cultured at $37^{\circ} \mathrm{C} ; 50 \mu \mathrm{L} 1 \mathrm{mM}$ isopropyl- $\beta-\mathrm{D}$ thiogalactopyranoside (IPTG) was added to induce the tac promoter. The E. colicells were harvested by centrifugation and PBS (200 $\mu \mathrm{L} /$ tube $)$ was added. After resuspension, cells were disrupted ultrasonically. Fifty microliters of lysate was taken as the whole cell lysate for later gel electrophoresis. The remaining amount was centrifuged for the separation of supernatant and pellet, regarded as soluble and insoluble protein samples, respectively. Then, $10 \mu \mathrm{L}$ of the whole cell lysate and soluble and insoluble protein samples were subjected to SDS-PAGE with $12.5 \%$ polyacrylamide gel and CBB-R250 staining.

\section{Western blotting}

For Western blotting analysis, proteins were transferred to a polyvinylidene fluoride membrane (Tiangen Biotech, China) with blocking buffer (1.5\% BSA, $20 \mathrm{mM}$ Tris- $\mathrm{HCl}$, $\mathrm{pH} 8.0,150 \mathrm{mM} \mathrm{NaCl}, 0.1 \%$ Tween 20) at $48 \mathrm{~mA}$ for 80 min. The membrane was incubated with primary antibody to Penta-His Antibody (Qiagen, Germany) 1:1000, at $4^{\circ} \mathrm{C}$ overnight, followed by incubation with secondary antibody of HRP-RabbitAnti-Mouse lgG(H+L) (Zymed, USA) 1:1000 for $1 \mathrm{~h}$ at room temperature, and proteins were then visualized using $1 \mathrm{~mL}$ TrueBlue peroxidase substrate (Kirkegaard and Perry, USA) for $1 \mathrm{~min}$.

\section{Bioinformatics of the cloned Der $\mathbf{f} 5$}

The open reading fragment (ORF) was determined using the ORF finder in the National Center for Biotechnology Information (NCBI) website. The amino acid sequence of Der $f 5$ was determined with Translate Tools on the ExPaSy webserver, its physicochemical properties were determined by the ProtParam tool, its signal peptide sequence by the SignalP 3.0 software, its hydrophilicity by ProtScale tools, the protein subcellular localization by CELLO v.2.5, and the secondary structure by the self-optimized prediction method with alignment (SOPMA) on the NPS@server.

The homology between the deduced amino acid sequence for the complete pre-pro form Der $f 5$ from China and other proteins was determined by comparing their sequences in all non-redundant GenBank CDS translations + PDB + SwissProt + PIR + PRF excluding environmental samples using BLASTp at the NCBI website. Based on the BLASTp search, the similar amino sequences of other mite species were chosen, i.e., Blomia tropicalis (Blo t 5, GenBank ABH06352), D. pteronyssinus (DER p 5, Gen- 
Bank P14004), Suidasia medanensis (Sui m 5, GenBank AAX34051), Lepidoglyphus destructor (Lep d 5, GenBank Q9U5P2), and Aleuroglyphus ovatus (Ale o 5, GenBank AAX34060). Sequences were matched with those of the group 5 allergens of these other mites using ClustalW 2.0 at the European Bioinformatics Institute (EBI) website and computed for similarity with the VECTOR NTI 9.0 software (IBI, USA). The polygenetic tree was constructed for the group 5 allergens of Der $f 5$ and the ohter mite species cited above using the Mega 4.0 software.

\section{Results}

\section{Construction of the recombinant plasmids pMD19-T-Der f 5}

After extraction of the total RNA of $D$. farinae, the Der $f$ 5 gene was synthesized by RT-PCR, and a strap of about 399 bp was present as determined by agarose gel electrophoresis. The PCR product was recovered and linked into the pMD19-T simple vector. Eight recombinant plasmids of pMD19-T-Der f 5 were subjected to enzyme digestion analysis, and the expected result was observed by $1.0 \%$ agarose gel electrophoresis (Figure 1). clone 2 was $97.8 \%$ homologous to the reference.

\section{Construction of plasmids pET28a-(+)-Der $f 5$ and its expression in E. coli BL21}

Based on sequence alignment, clone 2 was chosen for sub-cloning and expressed. After digestion of the recombinant plasmid pMD19-T-Der $f 5$ by both BamHI and Xhol, the recovered cDNA encoding Der $f 5$ was sub-cloned

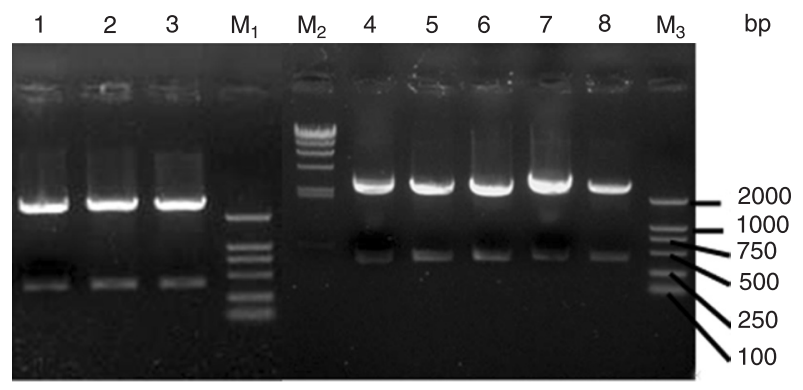

Figure 1. Restriction enzyme analysis of the recombinant plasmids pMD19-T-Der $\mathrm{f} 5$ by BamHI and Xhol. Lanes $M_{1}$ and $M_{3}=$ DL-2000 DNA marker; lane $M_{2}=\lambda$-Hind III DNA marker; lanes 1-8 $=$ recombinant plasmids digested with $\mathrm{BamHI}$ and Xhol.

\section{Nucleic acid sequence and analysis}

Five of the eight plasmids determined by restriction enzyme analysis were chosen and sequenced. After removal of the vector sequence and the added restriction sites, the length of the Der $f 5$ nucleic acid sequence was 399 bp from the start codon ATG to the stop codon TGA. Figure 2 shows the results of alignment of our sequencing for the five PCR clones, the reference (GenBank AY283283), and another published Der f 5 sequence (GenBank AB195581) by using the VECTOR NTI 9.0 software. Eight mutation positions were observed at 35,120 , 141, 159-161, 207, 215-217, 345 , and $368 \mathrm{bp}$. When these nucleotide sequences were translated into amino acid sequences, there were seven incompatible amino acid positions at $12,40,53,73,74,115$, and 123 (Figure 3). However, the nucleotide sequence for

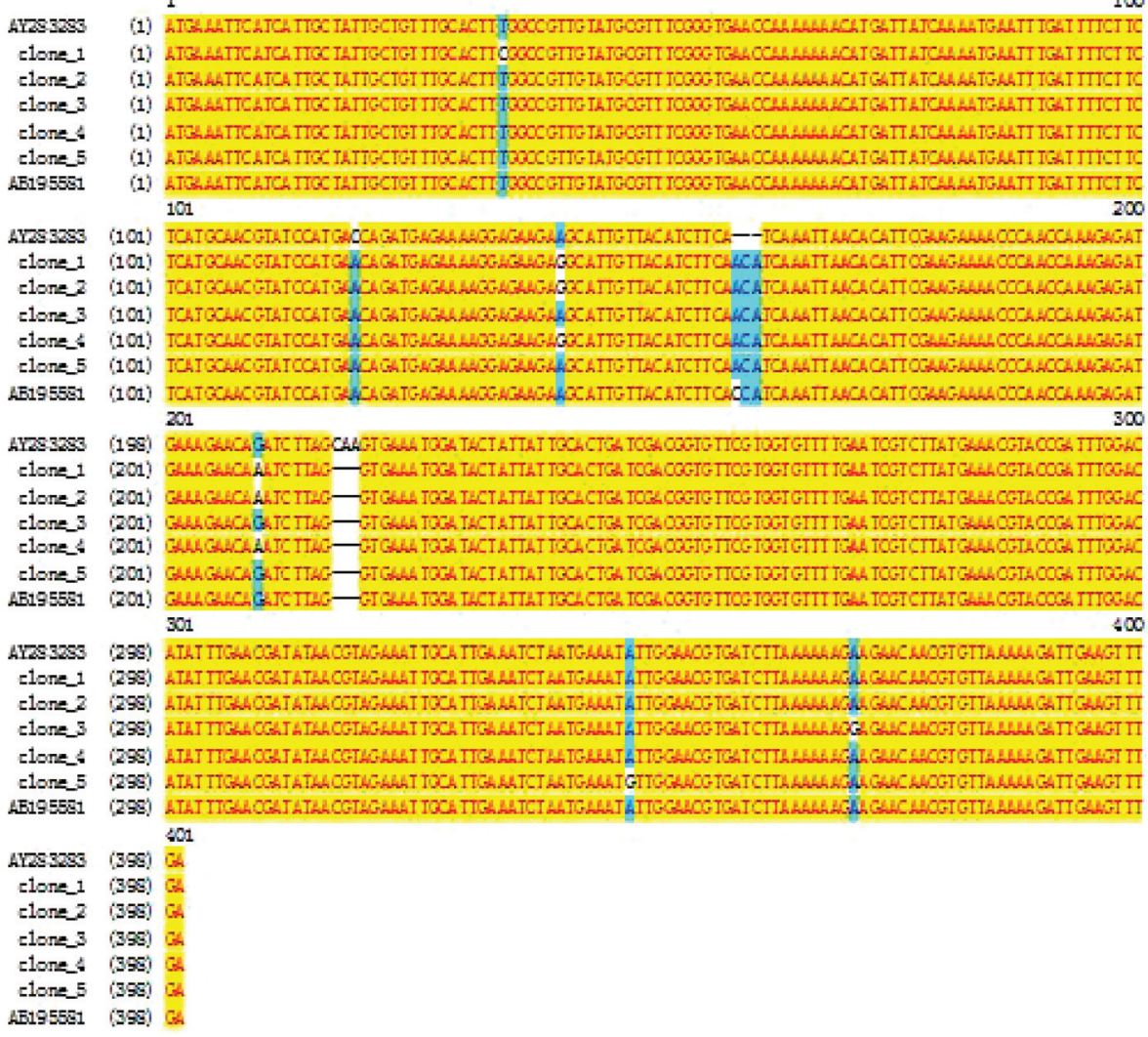

Figure 2. Nucleotide sequence alignment among different plasmids sequenced in this paper and the Der $f$ sequences published in GenBank by using the VECTOR NTI 9.0 software (IBI, USA). 
into the expression vector of pET28a-(+) and identified by restriction digestion. E. coli BL21 was then transformed with plasmid pET28a-(+)-Der $\mathrm{f} 5$ and the protein expressed was induced with IPTG at $37^{\circ} \mathrm{C}$ for $3 \mathrm{~h}$. Absorbance at $600 \mathrm{~nm}$ was measured for 0.562 and 1.259 before and after induction. A single specific band from SDS-PAGE and Western blotting (Figure $4 \mathrm{~A}$ and $\mathrm{B}$ ) was observed, corresponding to the expected molecular weight.

\section{Inferred amino acid} sequence and its structural and functional prediction

Five recombinant plasmids pMD19-T-Der $f 5$ were subjected to DNA sequencing, and the highest homology $(97.8 \%)$ with the reference sequence was observed in clone 2. One ORF was identified encoding a protein of 132 amino acids, 15525.1 Da, and theoretical isoelectric point ( $\mathrm{pl}$ ) of 5.62 . A signal peptide sequence of 1-19 amino acid residues was predicted by the SignalP 3.0 software. After removal of this leading sequence, the matured part of Der $f 5$ consisted of 113-amino acid residues (13604.03 Da, theoretical pl $=5.43$ ). The grand average of hydropathicity (GRAVY) of -0.803 indicated that this is a hydrophobic protein, confirmed by the ProtScale software. On the NPS@server, the secondary structure of the matured Der $f 5$ allergen was predicted using the SOPMA software, indicating that $90.3 \%$ (102 amino acids) of the protein was alpha helix, $0.88 \%$ (1 amino acid) extended strand, $0.88 \%$ beta turn, and $7.96 \%$ (9 amino acids) random coils. Its subcellular localization was predicted by the subCELlular LOcalization predictive system (CELLO) to be cytoplasmic (Table 1). The PROSCAN sequence search tool on the NPS@server indicated that the protein contains two casein kinase II phosphorylation sites (20-23 and 58-61 residues). NTI 9.0 software (IBI, USA).

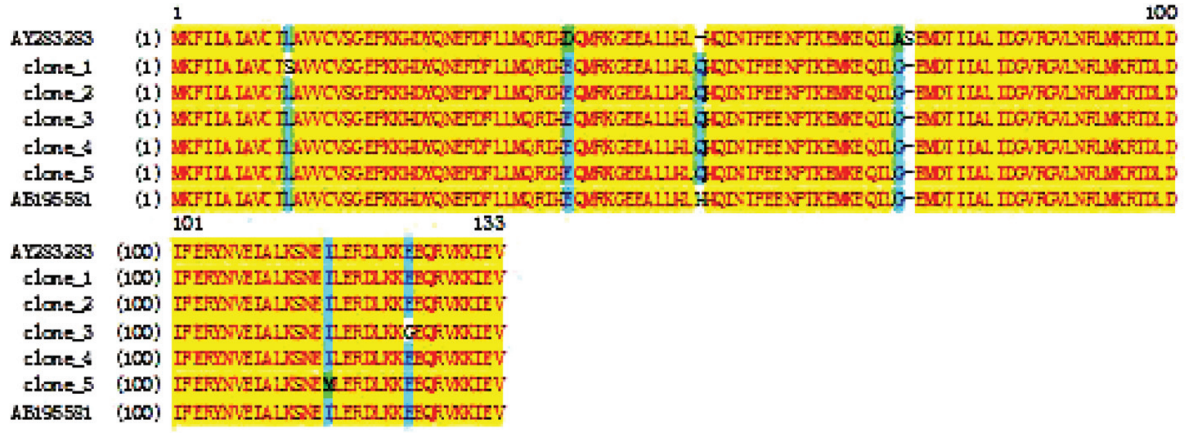

Figure 3. Results of alignment among amino acid sequences deduced from different plasmids sequenced in this paper and the Der $f 5$ sequences published in GenBank by using the VECTOR
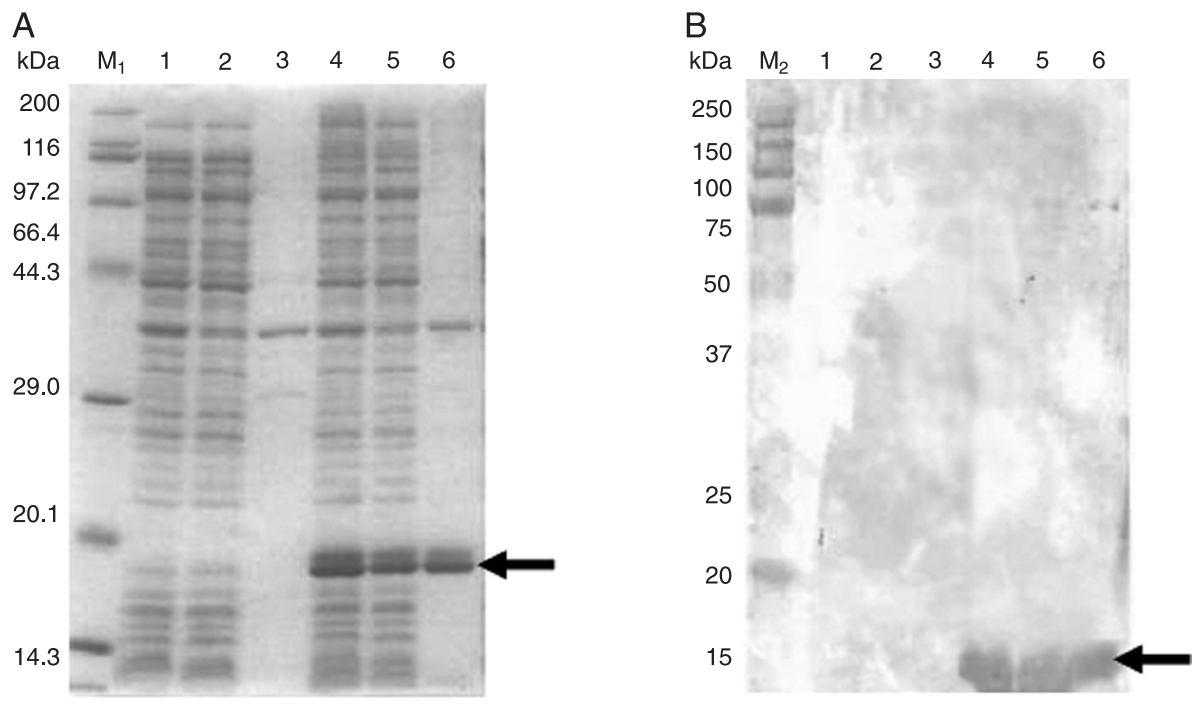

Figure 4. Expression of rDer $f 5$ in Escherichia coli BL21 cells. E. coli BL21 cells were transformed with either pET28a(+)-Der $f 5$ or empty vector pET28a(+) as control. A, SDS-PAGE analysis of the rDer $\mathrm{f} 5$ protein. Lane $M_{1}=$ TaKaRa protein marker (Broad); lane 1 = whole cell lysate of $E$. coli BL21 cells containing pET28a; lane 2 = supernatant of cells containing pET28a; lane 3 = pellet of cells containing $\mathrm{pET} 28 \mathrm{a}$; lane $4=$ whole cell lysate of $E$. coli BL21 cells containing $\mathrm{pET} 28 \mathrm{a}(+)-\operatorname{Der} \mathrm{f} 5$; lane $5=$ supernatant of cells containing pET28a(+)-Der $f 5$; lane $6=$ pellet of cells containing pET28a(+)-Der f 5. $B$, Western blotting analysis of the rDer $\mathrm{f} 5$ protein. Lane $M_{2}=$ Precision Plus Protein Standards; lane 1 = whole cell lysate of $E$. coli BL21 cells containing pET28a; lane $2=$ whole cell lysate of $E$. coli BL21 cells containing pET28a(+)-Der $f 5$. Arrows point to the band of rDer $f 5$.

\section{Analysis of amino acid sequence homology, alignment and molecular evolution}

The similarity of Derf5 and Derp 5, Blot5, Suim 5, and Lep d 5 was $79,48,53$, and $37 \%$, respectively (Figure 5, Table 2); Derf 5 and Derp 5 were clustered with $99 \%$ bootstrap, whereas Blo t 5 and Ale o 5 were clustered with 78\% (Figure 6).

\section{Discussion}

We cloned for the first time the full-length cDNA frag- 
ment encoding the group 5 allergen from $D$. farinae isolated in China. Five sequenced cDNA clones of Der $f 5$ were obtained simultaneously, and there still are eight mismatched nucleotides and seven incompatible amino acid residues in the complete pre-protein. This indicates that the Der $f 5$ allergen may have sequence polymorphism; only a single amino acid variation (at position 61, from alanine to aspartate) was presented in the amino acid sequence deduced from five cDNA clones of Der $p 5$ (17). More research should be done regarding the sequence polymorphisms of the group 5 dust mite allergens, which will be important for the development of peptide-based immunotherapeutic agents for mite-allergic patients.

Among 19 patients with positive skin tests to D. pteronyssinus extracts, 10 were positive to rDer p 5 (18), so the group 5 allergen should be incorporated into large-scale clinical trials. Therefore, we expressed the Der f 5 allergen in E. coli. A single specific band between 14 and $20 \mathrm{kDa}$ was observed after SDS-PAGE and Western blotting. The expression vector pET28a-(+), containing a nickel-binding 6-histidine, indicates that the recombinant product has an extra 6-histidine and the molecular weight of rDer $f 5$ should be lower than determined by SDS-PAGE and Western blotting in the present study.

When the complete sequence of Der $f 5$ cDNA was analyzed, one ORF with the full length of 399 bp was found included in the cDNA fragment, which was reckoned to encode a protein with 132-amino acid residues. Further analysis showed that there was a signal peptide sequence from 1- to 19-amino acid residues. After removal of this sequence,
Table 1. Result of the prediction of protein subcellular localization for Der $f$ 5 by CELLO v.2.5 (http://cello.life.nctu.edu.tw/).

\begin{tabular}{llc}
\hline Support vector machine & Localization & Reliability \\
\hline Amino acid composition & Cytoplasm & 0.357 \\
N-peptide composition & Cytoplasm & 0.993 \\
Partitioned sequence composition & Cytoplasm & 0.743 \\
Physicochemical composition & Cytoplasm & 0.937 \\
Neighboring sequence composition & Cytoplasm & 0.932 \\
CELLO prediction & & \\
& Cytoplasm & 3.962 \\
& Periplasmic & 0.359 \\
& Extracellular & 0.310 \\
& Inner membrane & 0.259 \\
& Outer membrane & 0.110 \\
\hline
\end{tabular}

Table 2. Analysis of similarity between Der $f 5$ and its homologous amino acid sequence of other mite species by the VECTOR NTI 9.0 software.

\begin{tabular}{lcccccc}
\hline Mite protein & \multicolumn{6}{c}{ Similarity (\%) } \\
\cline { 2 - 7 } & Ale o 5 & Blo t 5 & Der f 5 & Der p 5 & Sui m 5 & Lep d 5 \\
\hline Ale 0 5 & - & 52 & 37 & 37 & 50 & 32 \\
Blo t 5 & - & - & 48 & 44 & 50 & 38 \\
Der f 5 & - & - & - & 79 & 53 & 37 \\
Der p 5 & - & - & - & - & 52 & 32 \\
Sui m 5 & - & - & - & - & - & 39 \\
Lep d 5 & - & - & - & - & - & - \\
\hline
\end{tabular}

Ale o = Aleuroglyphus ovatus; Blo $\mathrm{t}=$ Blomia tropicalis; Der $\mathrm{f}=$ Dermatophagoides farinae; Der $\mathrm{p}=\mathrm{D}$. pteronyssinus; Sui $\mathrm{m}=$ Suidasia medanensis; Lep $\mathrm{d}=$ Lepidoglyphus destructor .

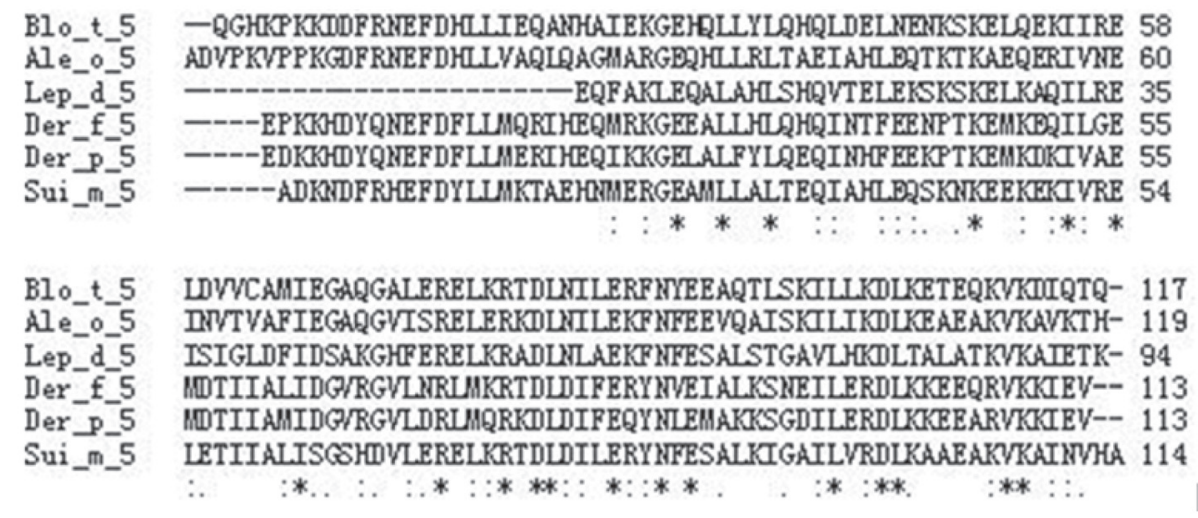

Figure 5. Alignment between Der $\mathrm{f} 5$ and its homologous amino acid sequence of other mite species by ClustalW2 (http://www.ebi.ac.uk/Tools/clustalw2/). Asterisks = residues or nucleotides in the column are identical in all sequences in the alignment; colons = conserved substitutions; periods $=$ semi-conserved substitutions. For abbreviations, see legend to Table 2. 
the active part of Der $f 5$ should consist of 113-amino acid residues with a calculated molecular mass of 13604.03 Da and theoretical pl of 5.43 - identical to previous reports that identified a partial cDNA clone of Der p 5, producing a recombinant polypeptide that reacted with a $14-\mathrm{kDa}$ band in mite extracts (19).

The general three-dimensional form of local segments of proteins (i.e., their secondary structure) is defined by patterns of hydrogen bonds between backbone amide and carboxyl groups. In the present study, the SOPMA software was used to predict the secondary structure of the matured part of Der $f 5$, and the analysis indicated that the allergen consisted of alpha helix (90.3\%), extended strand $(0.9 \%)$, beta turn $(0.9 \%)$, and random coils $(8.0 \%)$. Subsequently, the subcellular localization for Der $f 5$ showed that it should be cytoplasmic. However, this is a prediction, and its location must be confirmed experimentally.

Much of the published literature concerns allergens of house dust mites, which belong to the family Pyroglyphidae, especially $D$. pteronyssinus, $D$. farinae, and Euroglyphus maynei. Other mite species, referred to as 'storage mites', are also considered allergenic, but their study has been more limited (20). We utilized the deduced amino acid sequence of Der 5 from China in $\mathrm{NCBI}$ and similar amino sequences of other mites were obtained, including $D$. pteronyssinus (Pyroglyphidae), L. destructor (Glycyphagidae) and B. tropicalis (Echimyopodidae), S. medanensis (Suidasiidae), and A. ovatus (Acaridae). These mite species were considered to be important from an economic and sanitary perspective and are now being recognized as important contributors to

\section{References}

1. Milian E, Diaz AM. Allergy to house dust mites and asthma. $P R$ Health Sci J 2004; 23: 47-57.

2. Nadchatram M. House dust mites, our intimate associates. Trop Biomed 2005; 22: 23-37.

3. Bunnag $C$, Jareoncharsri $P$, Tantilipikorn $P$, Vichyanond $P$, Pawankar R. Epidemiology and current status of allergic rhinitis and asthma in Thailand - ARIAAsia-Pacific Workshop report. Asian Pac J Allergy Immunol 2009; 27: 79-86.

4. Thomas WR, Smith WA, Hales BJ, Mills KL, O'Brien RM. Characterization and immunobiology of house dust mite allergens. Int Arch Allergy Immunol 2002; 129: 1-18.

5. Thomas WR, Smith WA, Hales BJ. The allergenic specificities of the house dust mite. Chang Gung Med J 2004; 27: 563-569.

6. Dinakar C, Portnoy JM. Allergen immunotherapy in the prevention of asthma. Curr Opin Allergy Clin Immunol 2004; 4: 131-136.

7. Mauro M, Russello M, Alesina R, Sillano V, Alessandrini A, Dama A, et al. Safety and pharmacoeconomics of a cluster the allergen content of house dust $(4,5,20)$. They can cause occupational respiratory allergies in farmers and other occupationally exposed individuals (20). Many mite allergens have shown sequence homology and biological function similar to those described in Dermatophagoides spp (20). Sequence similarities in the present study were $79,48,53$, and $37 \%$ between Der $f 5$ and Der p 5, Blo t 5, Sui m5, and Lep d 5, respectively. In the phylogenetic tree constructed based on the group 5 allergens of the various mite species, Der f 5 and Der p 5 clustered with 99\% bootstrap. Interestingly, Blo t 5 and Ale o 5 clustered with $78 \%$, despite the fact that these two mites belong to different families. This illustrates the need for more molecular data in the study of relationships among mite species.

\section{Acknowledgments}

Research supported by the National Sciences Foundation of China (\#NSFC30060166 and \#NSFC81001330) and by the Jiangsu Provincial Health Department (Grants \#Z200914 and \#J200907). administration of mite immunotherapy compared to the traditional one. Eur Ann Allergy Clin Immunol 2006; 38: 31-34.

8. Zhang L, Wang C, Han D, Wang X, Zhao Y, Liu J. Comparative study of cluster and conventional immunotherapy schedules with Dermatophagoides pteronyssinus in the treatment of persistent allergic rhinitis. Int Arch Allergy Immunol 2009; 148: 161-169.

9. Jeong KY, Hongb CS, Yong TS. Recombinant allergens for diagnosis and immunotherapy of allergic disorders, with emphasis on cockroach allergy. Curr Protein Pept Sci 2006; 7: $57-71$.

10. Mothes N, Valenta R, Spitzauer S. Allergy testing: the role of recombinant allergens. Clin Chem Lab Med 2006; 44: 125-132.

11. Piboonpocanun S, Malainual N, Jirapongsananuruk O, Vichyanond P, Thomas WR. Genetic polymorphisms of major house dust mite allergens. Clin Exp Allergy 2006; 36: 510516.

12. Thomas WR, Heinrich TK, Smith WA, Hales BJ. Pyroglyphid 
house dust mite allergens. Protein Pept Lett 2007; 14: 943953.

13. Cui $Y$, Zhou P, Peng J, Peng M, Zhou Y, Lin Y, et al. Cloning, sequence analysis, and expression of cDNA coding for the major house dust mite allergen, Der f 1, in Escherichia coli. Braz J Med Biol Res 2008; 41: 380-388.

14. Cui YB, Cai HX, Li L, Zhou Y, Gao CX, Shi WH, et al. Cloning, sequence analysis and expression in $E$. coli of the group 3 allergen of Dermatophagoides farinae. Chin Med J 2009; 122: 2657-2661.

15. Yu-bao C, Zhou Y, Weihong S, Guifang M, Yang L, Yungang $W$. Cloning, expression, and analysis of the group 2 allergen from Dermatophagoides farinae from China. An Acad Bras Cienc 2010; 82: 941-951.

16. Cui YB, Cai HX, Zhou Y, Gao CX, Shi WH, Yu M, et al. Cloning, expression, and characterization of Der $\mathrm{f} 7$, an allergen of Dermatophagoides farinae from China. J Med Entomol
2010; 47: 868-876.

17. Lin $\mathrm{KL}$, Hsieh $\mathrm{KH}$, Thomas WR, Chiang BL, Chua KY. Characterization of Der $p \mathrm{~V}$ allergen, cDNA analysis, and IgE-mediated reactivity to the recombinant protein. J Allergy Clin Immunol 1994; 94: 989-996.

18. Simpson A, Green R, Custovic A, Woodcock A, Arruda LK, Chapman MD. Skin test reactivity to natural and recombinant Blomia and Dermatophagoides spp allergens among mite allergic patients in the UK. Allergy 2003; 58: 53-56.

19. Tovey ER, Johnson MC, Roche AL, Cobon GS, Baldo BA. Cloning and sequencing of a cDNA expressing a recombinant house dust mite protein that binds human $\lg E$ and corresponds to an important low molecular weight allergen. J Exp Med 1989; 170: 1457-1462.

20. Fernandez-Caldas E, Iraola V, Carnes J. Molecular and biochemical properties of storage mites (except Blomia species). Protein Pept Lett 2007; 14: 954-959. 